\title{
Acute conceptual disorganization in untreated first-episode psychosis: a combined magnetic resonance spectroscopy and diffusion imaging study of the cingulum
}

\author{
Yunzhi Pan, PhD; Kara Dempster, MD, MSc; Peter Jeon, MSc; Jean Théberge, PhD; \\ Ali R. Khan, PhD; Lena Palaniyappan, MD, PhD
}

\begin{abstract}
Background: Disorganized thinking is a core feature of acute psychotic episodes that is linked to social and vocational functioning. Several lines of evidence implicate disrupted cognitive control, excitatory overdrive and oxidative stress relating to the anterior cingulate cortex as mechanisms of conceptual disorganization (CD). We examined 3 candidate mechanistic markers related to CD in firstepisode psychosis: glutamate excess, cortical antioxidant (glutathione) status and the integrity of the cingulum bundle that connects regions implicated in cognitive control. Methods: We used fractional anisotropy maps from $7 \mathrm{~T}$ diffusion-weighted imaging to investigate the bilateral cingulum based on a probabilistic white matter atlas. We compared high CD, low CD and healthy control groups and performed probabilistic fibre tracking from the identified clusters (regions of interest within the cingulum) to the rest of the brain. We quantified glutamate and glutathione using magnetic resonance spectroscopy (MRS) in the dorsal anterior cingulate cortex. Results: We found a significant fractional anisotropy reduction in a cluster in the left cingulum in the high CD group compared to the low CD group (Cohen's $d=1.39 ; p<0.001$ ) and controls (Cohen's $d=0.86 ; p=0.009$ ). Glutamate levels did not vary among groups, but glutathione levels were higher in the high CD group than in the low CD group. We also found higher glutathione related to lower fractional anisotropy in the cingulum cluster in the high CD group. Limitations: The MRS measures of glutamine were highly uncertain, and MRS was acquired from a single voxel only. Conclusion: Acute CD relates to indicators of oxidative stress, as well as reduced white matter integrity of the cingulum, but not to MRI-based glutamatergic excess. We propose that both oxidative imbalance and structural dysconnectivity underlie acute disorganization.
\end{abstract}

\section{Introduction}

Conceptual disorganization (CD) refers to difficulties in the goal-directed sequencing of thoughts that manifest as circumstantial, illogical or tangential speech or weakened goal of thinking (loose associations) in patients with psychotic disorders such as schizophrenia. ${ }^{1}$ Among patients with schizophrenia, CD is an important contributor to reduced realworld functioning. ${ }^{2}$ It is closely linked to the construct of formal thought disorder (FTD), but unlike FTD, CD is a clinical interpretation that is not restricted to the speech domain and refers specifically to the positive (rather than the negative) dimension of disorganized thinking.

Based on the idea that CD relates to the aberrant shunting of cognitive processes, ${ }^{3,4}$ structural disconnection among brain regions has been investigated as the neural basis using diffusion tensor imaging (DTI). Viher and colleagues ${ }^{5}$ and Cavelti and colleagues ${ }^{6}$ found no association between white matter integrity and the severity of disorganization or FTD, but Bopp and colleagues ${ }^{7}$ noted more severe FTD in patients with reduced integrity of the cingulum tract. More recently, de Boer and colleagues ${ }^{8}$ found an association between computational linguistic indices relating to negative symptoms and white matter integrity in schizophrenia. This effect was stronger for the entire white matter structure, rather than for language-related tracts. ${ }^{8}$ These previous DTI studies were limited to patients with established illness ( $>2$ years) and with long exposure to antipsychotic medications that affected both DTI indices ${ }^{9}$ (but see Voineskos and colleagues ${ }^{10}$ ) and symptom severity scores. ${ }^{6,8}$ At present, the structural basis of acute $\mathrm{CD}$ in minimally treated patients in first-episode psychosis (FEP) is unknown. ${ }^{11,12}$

Correspondence to: L. Palaniyappan, Robarts Research Institute, Room 1232D, 1151 Richmond Street N, London, ON N6A 5B7; Ipalaniy@uwo.ca

Submitted Aug. 29, 2020; Revised Dec. 23, 2020; Accepted Jan. 19, 2021

DOI: $10.1503 /$ jpn.200167 
An experimental approach that provokes symptoms of acute $\mathrm{CD}$ is subanesthetic ketamine infusion in both healthy volunteers and patients with schizophrenia. ${ }^{13}$ Ketamine disrupts inhibitory interneuron function, resulting in the disinhibition of the excitatory glutamatergic outputs of the prefrontal cortex. Sustained disinhibition increases extracellular glutamate, resulting in oxidative stress. ${ }^{14}$ Glutathione (GSH), a neuronal antioxidant, is the primary defence against excitotoxicity in the human brain. ${ }^{15}$ Subgroups of patients with psychoses and higher levels of CD show elevated peripheral markers of oxidative stress. ${ }^{16-18}$ Reduced GSH and glutamate have been reported in the anterior cingulate cortex (ACC) in several magnetic resonance spectroscopy (MRS) studies in schizophrenia. ${ }^{19,20}$ However, despite the relevance of the ketamine model to $\mathrm{CD}_{1}^{11}$ the role of brain glutamate and GSH in CD remains unstudied to date.

In this study, we focused on the cingulum (a frontal association bundle) and the dorsal ACC related to acute CD for 3 important reasons. First, the structural integrity of the cingulum has repeatedly been observed to be abnormal in psychosis, 7,21,22 and this disruption has been linked to the positive symptoms of schizophrenia. ${ }^{23-26}$ Second, cognitive control function is presumed to be disrupted in patients with high CD. ${ }^{27}$ Cingulum integrity underlies several aspects of the cognitive control dysfunction seen in schizophrenia..$^{28,29}$ The cingulum is the primary association tract that connects the dorsal ACC with other sites that have been implicated in cognitive control, such as the precuneus. ${ }^{30}$ Third, in previous structural studies we reported volumetric abnormalities in networks anchored on the dorsal ACC in relation to thought disorder. ${ }^{31}$ In particular, disorganized thinking was associated with structural variations in the cognitive control regions, more than in language regions. ${ }^{32}$ Abnormalities in the structural integrity of the cingulum have been linked to $\mathrm{CD}$ by Bopp and colleagues. ${ }^{7}$ Finally, given its protracted development compared to other long bundles, ${ }^{33}$ the cingulum is considered to be particularly vulnerable to maturational aberrations relevant for schizophrenia. Markers of oxidative stress, such as reduced GSH in the ACC, relate to the maturational integrity of the cingulum bundle. ${ }^{34}$

In the current study, we bring together 2 candidate mechanistic markers related to acute CD in FEP: glutamate excess and antioxidant (GSH) status in the ACC using MRS; and the structural integrity of the cingulum based on fractional anisotropy (FA), using DTI. We also investigated the relationship between these mechanistic features in patients with untreated FEP and a median antipsychotic medication exposure of less than 3 days. This study is part of an ongoing preregistered observational trial (NCT02882204) investigating the pathophysiology of FTD in the early stages of schizophrenia with a focus on medial prefrontal connectivity, glutamatergic excess and GSH abnormalities.

\section{Methods}

\section{Participants}

Patients with FEP (31 with schizophrenia; 9 with schizoaffective/schizophreniform psychosis) between the ages of 15 and 39 years were recruited from the Prevention and Early Intervention for Psychosis Program at London Health Sciences Centre in London, Ontario, Canada, between April 2017 and July 2019. The program uses an assertive casemanagement model to provide assessment and treatment to individuals experiencing FEP.

In total, all patients and 25 healthy controls had scans of adequate quality (without obvious artifact and visible structural damage) for DTI analysis. Patients included in the study were experiencing FEP and had received antipsychotic treatment for less than 14 days. Both inpatients and outpatients were eligible to participate if they were able to provide informed consent and safely participate in the MRI protocol. All participants received a consensus diagnosis from 3 psychiatrists (L.P./K.D. and the primary treatment provider) after approximately 6 months, on the basis of the best estimate procedure (as described by Leckman and colleagues ${ }^{35}$ ) and the Structured Clinical Interview for DSM-5. ${ }^{36}$ After the 6-month consensus diagnosis, participants who met the criteria for bipolar disorder with psychotic features, major depressive disorder with psychotic features or suspected drug-induced psychoses were excluded from further analyses. Care was provided as usual to the study participants through their psychiatrist and other allied health members of the Prevention and Early Intervention for Psychosis Program. Antipsychotic medications were chosen by the treating psychiatrist in collaboration with the patient and/or their substitute decision-maker. Patients were offered the option of treatment with a long-acting injectable at the earliest opportunity, ${ }^{37}$ in accordance with current national guidelines for the treatment of FEP.

Recruitment of healthy controls $(n=25)$ was carried out through posters that advertised the opportunity to participate in a neuroimaging study to track the outcomes of FEP. Healthy controls had no personal history of mental illness and no first-degree relatives with a history of psychotic disorders. Group matching with the FEP cohort for age, sex and parental education was maintained.

Exclusion criteria for both the FEP and healthy control groups were as follows: meeting the criteria for a substance use disorder in the past year, according to the DSM-5; ${ }^{36}$ a history of a major head injury (leading to a substantial period of unconsciousness or seizures); a substantial, uncontrolled medical illness; or any MRI scan contraindications.

All potential study participants provided written, informed consent before participation, as outlined by the Western University Health Sciences Research Ethics Board. The sample reported here partially overlaps with those of 2 of our previous studies. ${ }^{38,39}$

\section{Symptom assessment}

We assessed the severity of symptoms in patients using the 8-item Positive and Negative Syndrome Scale (PANSS-8), ${ }^{40}$ which measures the severity of positive and negative symptoms. We also used the Social and Occupational Functioning Assessment Scale (SOFAS) to assess the overall level of functioning. ${ }^{41}$ We used a modified digit symbol substitution task (DSST) to quantify processing speed, a cognitive function 
that shows the most prominent reduction among patients with psychosis. ${ }^{42}$ The written and oral items on the DSST were scored separately, and we used the mean scores from these items for assessment, as in our previous study. ${ }^{43}$

We used ultra-high-resolution (7 $\mathrm{T})$ diffusion-weighted imaging in 40 patients with FEP and 25 controls to investigate the bilateral cingulum, defined according to the Johns Hopkins University white matter atlas. ${ }^{44}$ Based on the CD scores from the PANSS-8, we divided the patients into 2 groups: low CD (P2 = 1-3) and high CD (P2 = 4-6; see Appendix 1, available at jpn.ca/200167-a1, for more on this rationale). We chose this cut-off to distinguish between those with clinically severe symptoms and those with minimal symptoms, as recommended by the Remission Working Group..$^{45}$ This cut-off has also recently been employed to distinguish patients with or without FTD and language dysfunction. ${ }^{46}$ These 2 groups were not different in terms of the severity of other positive or negative symptoms. We applied tract-based spatial statistics (TBSS) to compare FA values using FSL software ${ }^{47}$ with 5000-permutation correction within the selected tract. We used $F$ tests for group comparisons.

\section{MRI data acquisition}

We acquired all magnetic resonance images on a $7.0 \mathrm{~T}$ Siemens Magnetom Terra MRI scanner using an 8-channel transmit/32-channel receive head coil at the Centre for Functional and Metabolic Mapping, Robarts Research Institute, Western University, London, Ontario, Canada. We acquired DTI using 64 diffusion-weighted volumes ( $\mathrm{b}$ value $=1000 \mathrm{~s} / \mathrm{mm}^{2}$ ) and an uneighted volume, with a 2-dimensional, spin-echo, echo-planar imaging sequence (matrix size $=104 \times 104 ; 72$ slices; voxel resolution $2 \times 2 \times$ $2 \mathrm{~mm}$; repetition time $5100 \mathrm{~ms}$; echo time $50.2 \mathrm{~ms}$; generalized autocalibrating partial parallel acquisition 3; multiband 3; phase partial Fourier 6/8).

\section{MRI preprocessing}

We processed diffusion-weighted images using an opensource, containerized application called "prepdwi" (www. github.com/khanlab/prepdwi), which uses the BIDS ${ }^{48}$ and BIDS Apps ${ }^{49}$ standards to perform standardized preprocessing and fitting. We first preprocessed diffusion-weighted MRI data with denoising using a local PCA method with "dwidenoise" from mrtrix ${ }^{50}$ and correction of ringing artifacts with the "unring" tool. ${ }^{51}$ We corrected eddy current and susceptibility-based distortions using "topup"52 and "eddy"53 based on FSL, with the "-repol" option enabled for outlier replacement. ${ }^{54}$ We performed within-subject rigid registration of the corrected diffusion-weighted imaging volume and the $T_{1}$-weighted volume using block-matching ${ }^{55}$ to bring the diffusion-weighted images into the same space as the $T_{1}$-weighted images. We corrected gradient nonlinearities using vendor-provided spherical harmonic coefficients for the gradient system (AC84), applied with the "gradient_ unwarp" tool ${ }^{56}$ to generate a nonlinear transformation, which was composed with the $T_{1}$-weighted linear transformation to resample the diffusion-weighted images into the corrected $T_{1}$ weighted space in a single step. We used modulation with the determinant of the Jacobian matrix of the unwarping to correct for intensity differences in the magnitude images because of gradient nonlinearities. We then used preprocessed diffusion-weighted images in the $T_{1}$-weighted space to estimate diffusion tensor metrics using "dtifit."57 All participants' FA images were first nonlinearly aligned to an FMRIB58_FA standard brain image using nonlinear registration and then used to create a mean FA skeleton representing the centres of all tracts common to all participants (threshold of 0.2). After that, each participant's aligned FA was projected onto this skeleton and fed into voxel-wise comparison. The voxels with significant group differences were labelled using the Johns Hopkins University white matter atlas ${ }^{44}$ and enlarged using the "TBSS_fill" tool (https://fsl.fmrib.ox.ac.uk/fsl/fslwiki/ TBSS/UserGuide?highlight=\%28TBSS_fill\%29) for illustrative purposes. In addition to FA, we undertook post hoc comparisons of radial diffusivity (reflecting myelin content), axial diffusivity (reflecting axonal integrity) and mode of anisotropy (reflecting fibre organization) for the regions that showed significant FA abnormalities.

We performed fibre tracking analysis using Bedpostx (based on FSL) for local modelling of diffusion parameters and the Probtrackx (https://fsl.fmrib.ox.ac.uk/fsl/fslwiki/ FDT) for tractography and connectivity-based segmentation (Appendix 1, Figure S1). Probabilistic fibre tracking is performed from the seed region that shows a difference in the group comparison to the whole brain of fibres in the native space, with parameters as follows: way total number $=70000$; number of samples (number of individual pathways that are drawn through the probability distributions on principle fibre direction) $=5000$; curvature threshold $=0.2$; maximum number of steps $=2000$; step length $=0.5 \mathrm{~mm}$; fibthresh (subsidiary fibre volume threshold) $=0.01$. We performed 2 -stage registration, initially with FLIRT for linear registration and then with FNIRT for nonlinear registration, both using FSL. We extracted the mean FA value and the volume of the traced fibres from each participant for further analysis.

\section{Metabolites assessment}

As described in our previous study, ${ }^{58}$ we measured metabolites (glutamate, GSH, glutamine) using a Siemens Magnetom 7.0 T MRI (Siemens Healthineers) using an 8-channel transmit/32-channel receive, head-only radiofrequency coil at the Centre for Metabolic Mapping at Western University. A $2.0 \times 2.0 \times 2.0 \mathrm{~cm}\left(8 \mathrm{~cm}^{3}\right)$ proton magnetic resonance spectroscopy $\left({ }^{1} \mathrm{H}-\mathrm{MRS}\right)$ voxel was placed in the bilateral dorsal ACC using a 2-dimensional anatomic imaging sequence in the sagittal direction (37 slices; repetition time $8000 \mathrm{~ms}$; echo time $70 \mathrm{~ms}$; flip angle $[\alpha] 120^{\circ}$; thickness $3.5 \mathrm{~mm}$; field of view $240 \times 191 \mathrm{~mm}$ ). We acquired a total of 32 channel-combined, water-suppressed spectral averages using the semi-LASER ${ }^{1} \mathrm{H}$-MRS pulse sequence (repetition time $7500 \mathrm{~ms}$, echo time $100 \mathrm{~ms}$ ) during each scan session; participants were at rest and asked to stare at a white cross on a screen for 4 minutes. Water suppression was 
achieved using the VAPOUR preparation sequence, and water-unsuppressed spectra were acquired for spectral postprocessing (lineshape deconvolution) and quantification (water amplitude as concentration reference). Each of the 32 spectral acquisitions was corrected for frequency and phase drifts as described in Near et $\mathrm{al}^{59}$ before averaging. Metabolite concentrations were corrected for grey and white matter volumes using previously described methods ${ }^{60}$ in Barstool. ${ }^{61}$ Water-subtracted spectra were modelled using the fitMAN a priori-based minimization algorithm and a quantification template that included 17 metabolite spectral signatures derived from simulation. ${ }^{61}$ Importantly, we used a long echo time acquisition, optimized for GSH and glutamate signals (in line with the trial preregistration) with minimal macromolecular interference, as per Wong and colleagues. ${ }^{61}$ We inspected all spectra visually for quality, and included only metabolites with Cramer-Rao lower bounds (CRLB) of less than $10 \%$ in the analyses. An example of representation of fit is provided in Figure 1. For glutamine, the mean CRLB of over $20 \%$ for both FEP and healthy control groups did not meet our rigorous CRLB cut-off of less than $10 \%$, indicating higher uncertainty in its quantification compared with glutamate and GSH spectra. We have reported the results for glutamine in Appendix 1.

We previously examined the overall resting functional MRI (fMRI) correlates of CD from an overlapping sample; this has been reported elsewhere. ${ }^{62}$

\section{Statistical analyses}

We carried out statistical analyses of participant demographic and clinical characteristics and parameters of imaging using SPSS 22.0 (IBM). We made group comparisons of the variables using independent-samples $t$ tests or

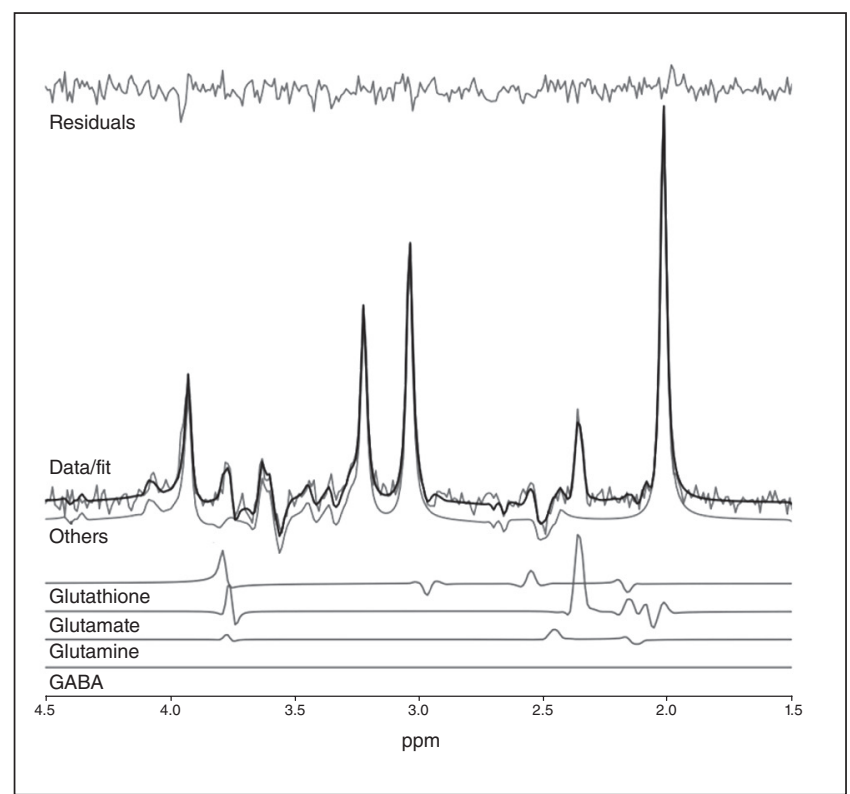

Fig. 1: Example of representation of fit for metabolites. GABA = $\gamma$-aminobutyric acid.
$F$ tests for continuous variables; Kruskal-Wallis $H$ tests for group comparison of ordinal items; or $\chi^{2}$ tests for proportions, as appropriate. We performed voxel-wise group comparisons and within-group comparisons on the mean FA skeleton in the framework of the generalized linear model using the Randomize tool in FSL. ${ }^{63}$ All analyses were conducted with 5000 permutations. We set the significance level at $p<0.05$ with threshold-free cluster enhancement correction ${ }^{64}$ and family-wise error rate correction for multiple comparisons.

\section{Results}

\section{Demographic and clinical characteristics}

All patients had acute psychosis, were minimally treated and had significant social and occupational dysfunction at the time of scanning. The demographic and clinical characteristics of the sample are presented in Table 1 . The 2 patient groups did not differ in terms of age, sex, socioeconomic status, duration of untreated illness in days, exposure to antipsychotic medication in days, total daily dose of antipsychotic medication or number of patients with schizophrenia in either group (diagnosed 6 months after the initial presentation). Across the 3 groups, we observed significant differences in SOFAS and DSST scores, confirmed by analysis of variance.

\section{Group differences in white matter integrity}

In the voxel-wise $F$ test, we observed a cluster (MNI coordinates $x, y, z=-8,-1,34)$ with a significant difference in FA ( $p=0.036$, corrected) among the 3 groups in the left cingulum (Figure 2A). We extracted mean FA, axial diffusivity, radial diffusivity and mode of anisotropy value in this cluster (Figure 2B). The high CD group showed lower FA than the low CD group (Cohen's $d=1.39, p<0.001$ ) and the healthy controls (Cohen's $d=0.86, p=0.009$ ). Moreover, the high CD group had lower axial diffusivity than the low CD group (Cohen's $d=0.84, p=0.021$ ). Interestingly, the low CD group had significantly higher axial diffusivity than the healthy controls (Cohen's $d=0.63, p=0.025$ ), indicating an aberrant increase in the axonal integrity of the cingulum in patients with lower levels of CD. We did not find any significant difference in radial diffusivity or mode of anisotropy value among the 3 groups.

Upon fibre tracking from the seed region to the whole brain, the high CD group showed significantly less volume of all fibres than the low CD group (Cohen's $d=0.69, p=0.030$ ) and healthy control group (Cohen's $d=0.81, p=0.019$ ). The mean FA of the tracked fibres did not differ among the 3 groups, indicating localized changes to the cingulum bundle (independent $t$ tests, $p=0.11$ to 0.76 ), although the mean FA of the tracked fibres was lower in patients with lower DSST scores $(r=0.381, p=0.015)$. We found no significant relationship between FA and DSST in the healthy control group $(r=-0.152, p=0.47)$. The volume of all fibres was negatively correlated with P2 value $(r=-0.264, p=0.034)$. 
Table 1: Demographic and clinical characteristics*

\begin{tabular}{|c|c|c|c|c|c|}
\hline \multirow[b]{2}{*}{ Measure } & \multicolumn{3}{|c|}{ Group $†$} & \multicolumn{2}{|c|}{ Group comparison } \\
\hline & High CD & Low CD & Healthy controls & $F / t / \chi^{2}$ & $p$ value \\
\hline \multicolumn{6}{|l|}{ Demographic information } \\
\hline Participants, $n$ & 16 & 24 & 25 & & \\
\hline Age, yr & $21.81 \pm 3.17$ & $23.71 \pm 5.43$ & $22.12 \pm 3.54$ & 1.237 & 0.30 \\
\hline $\mathrm{M} / \mathrm{F}, n$ & $13 / 3$ & $18 / 6$ & $14 / 11$ & 3.51 & 0.17 \\
\hline Exposure to antipsychotic medication, $d$ & $9.75 \pm 16.46$ & $12.57 \pm 12.42$ & - & 0.609 & 0.55 \\
\hline Duration of untreated illness, $d$ & $357.29 \pm 412.90$ & $331.64 \pm 619.81$ & - & -0.136 & 0.89 \\
\hline Total DDD of antipsychotics & $1.80 \pm 1.99$ & $1.99 \pm 4.58$ & - & -0.178 & 0.86 \\
\hline Duration of exposure to antipsychotics, $d$ & $3.87 \pm 3.78$ & $2.19 \pm 2.97$ & - & 2.210 & 0.15 \\
\hline Diagnosis of schizophrenia/other psychosis & $11 / 5$ & $20 / 4$ & - & 1.17 & 0.28 \\
\hline \multicolumn{6}{|l|}{ Clinical and cognitive assessments } \\
\hline \multicolumn{6}{|l|}{ PANSS-8 scores $\ddagger$} \\
\hline Conceptual disorganization (P2)§ & $4.69 \pm 0.79$ & $1.63 \pm 0.87$ & - & 30.09 & $<0.001$ \\
\hline Lack of spontaneity and flow of conversation (N6)§ & $2.06 \pm 1.44$ & $2.13 \pm 1.33$ & - & 0.044 & 0.84 \\
\hline Total positive component $(\mathrm{P} 1+\mathrm{P} 3+\mathrm{G} 9) \S$ & $14.56 \pm 2.99$ & $12.67 \pm 2.35$ & - & 4.018 & 0.05 \\
\hline Blunted affect and social withdrawal $(\mathrm{N} 1+\mathrm{N} 4) \S$ & $4.56 \pm 3.41$ & $5.58 \pm 3.12$ & - & 1.710 & 0.19 \\
\hline SOFAS score & $33.79 \pm 10.51$ & $42.26 \pm 13.60$ & - & 4.966 & 0.032 \\
\hline Modified DSST score & $51.94 \pm 15.10$ & $53.29 \pm 13.40$ & $68.74 \pm 11.09$ & 11.667 & $<0.001 \emptyset$ \\
\hline \multicolumn{6}{|l|}{ Metabolic assessments } \\
\hline Glutamate, $\mathrm{mM}$ & $6.57 \pm 0.75$ & $6.86 \pm 1.45$ & $6.69 \pm 0.81$ & 0.267 & 0.77 \\
\hline Glutathione, $\mathrm{mM}$ & $1.83 \pm 0.38$ & $1.61 \pm 0.36$ & $1.48 \pm 0.25$ & 3.894 & $0.027^{\star \star}$ \\
\hline Glutamate:glutathione & $3.69 \pm 0.59$ & $4.36 \pm 0.86$ & $4.58 \pm 0.47$ & 5.977 & $0.005^{\star *}$ \\
\hline CRLB glutamate & $3.67 \pm 1.39$ & $3.68 \pm 1.03$ & $3.24 \pm 0.99$ & 0.931 & 0.40 \\
\hline CRLB glutathione & $9.85 \pm 3.83$ & $11.18 \pm 4.02$ & $10.59 \pm 4.36$ & 0.383 & 0.68 \\
\hline \multicolumn{6}{|c|}{$\begin{array}{l}\mathrm{CD}=\text { conceptual disorganization; CRLB }=\text { Cramér-Rao lower bound; DDD = defined daily dose; DSST = digit symbol substitution tes } \\
\text { PANSS-8 = 8-item Positive and Negative Syndrome Scale; SOFAS = Social and Occupational Functioning Assessment Scale. } \\
\text { *Participants included in the final analysis. Number of participants with MRS data: healthy controls }=18, \text { high } C D=11, \text { low } C D=21 . \\
\text { †Values are presented as mean } \pm \text { standard deviation, unless otherwise indicated. } \\
\text { fLack of spontaneity and flow of conversation (N6) means a participant's lack of initiative, spontaneous and fluent conversation. } \\
\text { §Kruskal-Wallis } H \text { test. } \\
\text { १High } C D=\text { low } C D<\text { healthy controls. } \\
{ }_{\star * *} \mathrm{HIgh} C D>\text { low } C D=\text { healthy controls. }\end{array}$} \\
\hline
\end{tabular}

\section{Group differences in MRS metabolite measures}

The $F$ tests showed no significant difference in glutamate levels among the 3 groups, but a significant difference in GSH levels ( $F=3.894, p=0.027$; Figure 3 ). The high $C D$ group had higher GSH levels than the healthy controls (Cohen's $d=$ $1.096, p=0.008)$. Given the suspected differences in the neuronal versus astrocytic origin of the glutamate and GSH signals, respectively (see Duarte and $\mathrm{Xin}^{65}$ and Harris and colleagues ${ }^{66}$ for a review), we calculated the glutamate-GSH ratio as a proxy indicator of relative neuronal density. We found that the high CD group had a significantly lower ratio (driven by higher GSH concentration) than the low CD group (Cohen's $d$ $=0.912, p=0.011$ ) and the healthy controls (Cohen's $d=1.663$, $p=0.001$ ) indicating a possible increase in metabolite signals of astrocytic origin (GSH) compared to those of neuronal origin (glutamate) in relation to disorganization.

\section{Relationship between cingulum microstructure and MRS glutamate and GSH}

Axial diffusivity of the cingulum cluster was negatively related to glutamate $\left(r_{16}=-0.586, p=0.009\right)$ and GSH $\left(r_{16}=-0.523, p=0.026\right)$ in healthy controls, but only to GSH $\left(r_{9}=-0.797, p=0.003\right)$ in the high CD group. We found no DTI-MRS correlations in the low CD group (all $p>0.1$ ).

To determine if the observed relationship between DTI metrics, GSH and P2 score on the PANSS-8 scale (conceptual disorganization) was driven by other symptom differences or antipsychotic exposure status, we related GSH concentration, axial diffusivity and fibre volume to negative symptom burden, positive symptom burden, total defined daily dose and total medication days. None of these correlations was significant ( $r=-0.16$ to $0.2 ; p=0.27$ to 0.98$)$. Cumulative medication exposure (defined daily dose $\times$ days) did not differ between the low CD and high CD groups $(t=-0.178, p=0.86)$; we also found no differences between these groups in terms of the total number of antipsychotic exposure days $(t=1.486, p=$ 0.15). To determine if the observed relationship between DTI metrics, GSH and P2 score was an artifact of binarization, we ran a Spearman's correlation between GSH concentration, axial diffusivity, and fibre volume and P2 scores in all participants. We noted that P2 scores were negatively correlated to the volume of fibres from the seed region to whole brain $(r=-0.264, p=0.034)$, but we found no significant correlation between P2 score, axial diffusivity and GSH ( $p>0.1)$. 
A

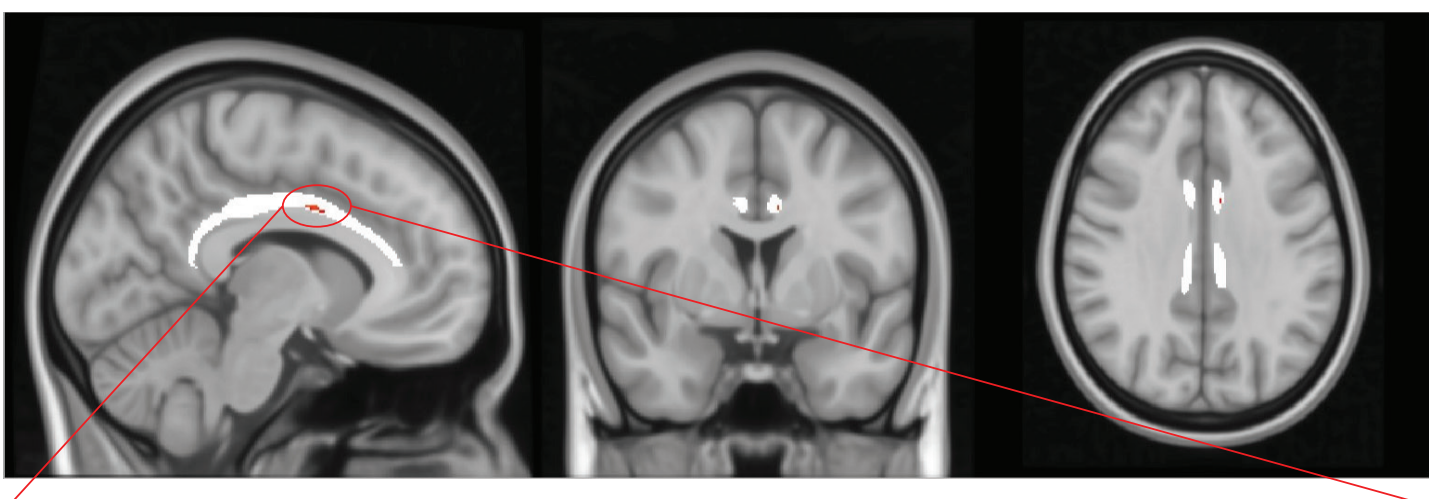

B
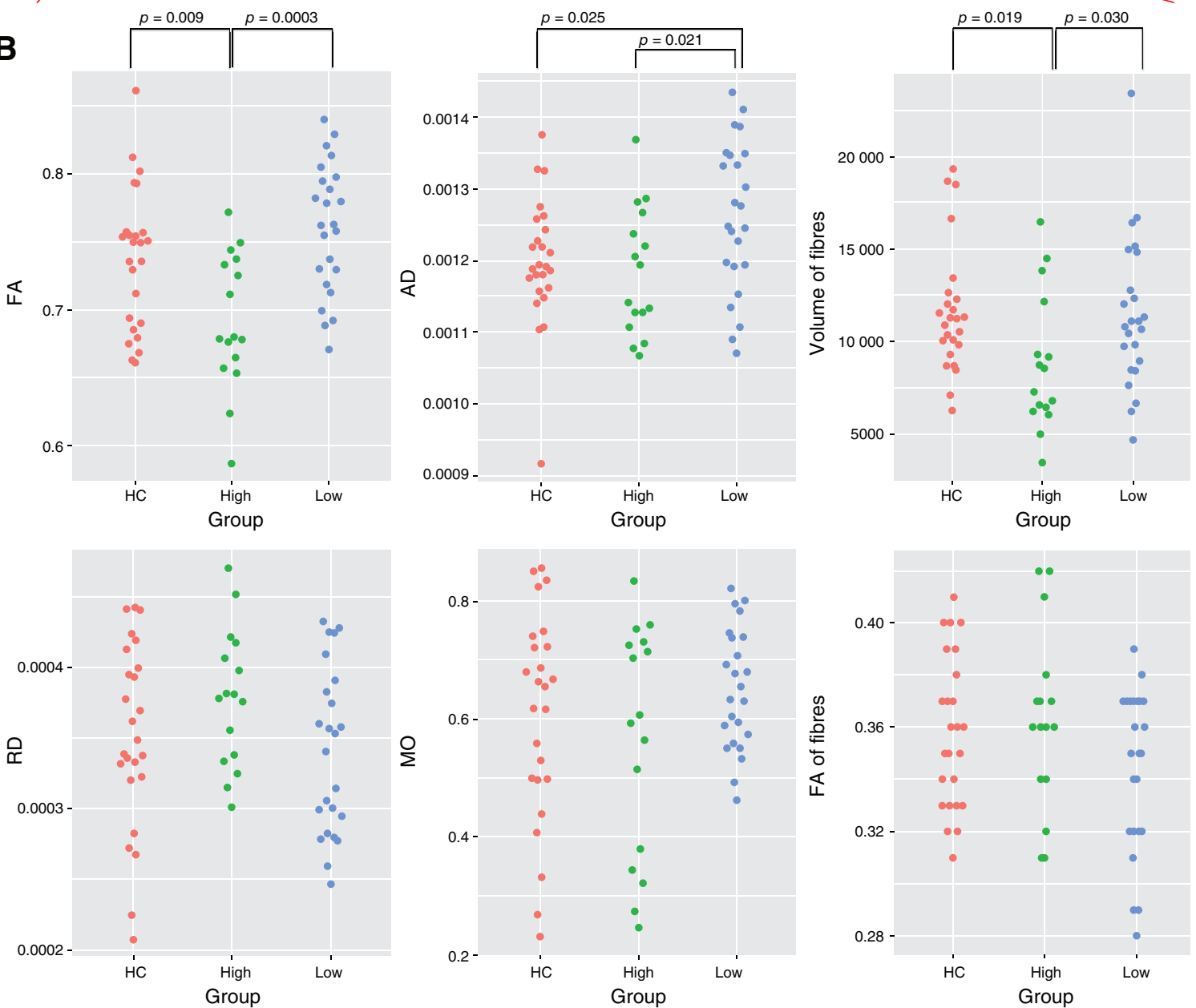

Fig. 2: A small part of the cingulum tract showed reduced FA and $A D$ in the group with high conceptual disorganization. (A) Results of $F$ tests of tract-based spatial statistics among 3 groups in the bilateral cingulum tract with permutation testing $(n=5000)$. (B) Values for $F A, R D, A D, M O$, total volume of fibres and FA of fibres tracked from the centroid of the seed region, identified in tract-based spatial statistics to the rest of the brain. $A D=$ axial diffusivity; $F A=$ fractional anisotropy; $H C=$ healthy controls; $M O=$ mode of anisotropy; $R D=$ radial diffusivity. 

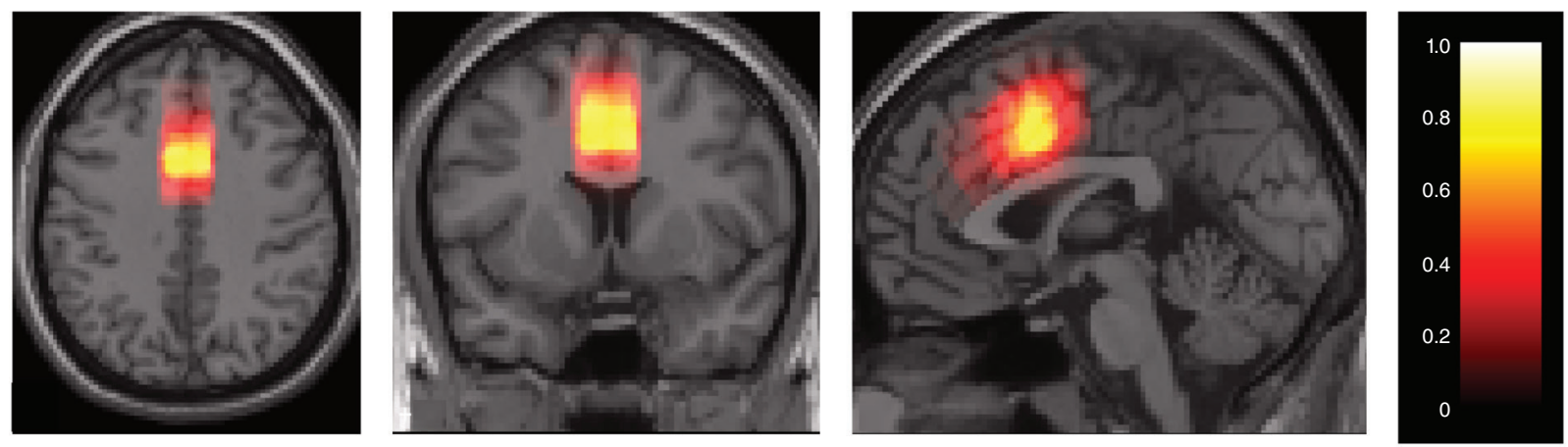

Fig. 3: Probabilistic map of magnetic resonance spectroscopy cingulate cortex voxel placement. Colour bar indicates the probability of the placed voxel across all participants (0 to 1$)$.

\section{Discussion}

To our knowledge, this is the first study to link CD to markers of excitotoxicity (glutamate), oxidative stress (GSH) and white matter integrity in the cingulum in FEP. We report 3 major observations: the presence of severe acute CD in FEP is associated with reduced white matter integrity of the cingulum, characterized by reduced axial diffusivity and decreased volume of fibres in the cingulum tract; it is also associated with increased GSH concentration in the dorsal ACC; and it is not associated with increased glutamate concentration. In addition, higher cingulum integrity was related to lower glutamate and GSH levels in healthy controls. Our findings suggest that the severity of $C D$ in untreated FEP may be related to markers of oxidative stress and white matter damage, but not to glutamate deficits as indexed using MRS.

Our observation of reduced white matter integrity of the cingulum in patients with high $C D$ was consistent with the findings of Bopp and colleagues, ${ }^{7}$ who systematically studied white matter integrity in relation to persistent disorganization. Reduced white matter integrity in the cingulum is a well-replicated finding in psychotic disorders, ${ }^{22,67-69}$ although heterogeneous changes are often reported at the whole brain level. ${ }^{70}$ The cingulum is a large body of fibre connecting multiple midline brain regions that play a crucial role in selfrelated processing (precuneus, ventromedial prefrontal cortex and medial temporal cortex). ${ }^{71}$ Individual differences in cingulum microstructure be linked to cognitive control measures in healthy individuals, ${ }^{30}$ as well as in patients with schizophrenia. ${ }^{72,73}$ Disrupted connectivity of the cingulum reported here supports the notion that the severity of $C D$ relates to disrupted domain-general control aspects of information processing in schizophrenia. ${ }^{32}$

We found increased GSH levels in patients with a high degree of CD in acute FEP, despite the normal levels of glutamate in the ACC. This association was specific to CD, as nei- ther negative symptom burden nor positive symptom severity was related to GSH concentrations in this sample. In an overlapping sample, we previously noted that people with FEP and higher GSH were likely to respond more quickly to treatment, despite their higher symptom burden. Interestingly, among adolescents at risk for psychosis, increased GSH levels in the ACC have been linked to a higher degree of disorganization. ${ }^{74}$ Taken together, these results suggest that an acute oxidative stress response associated with disrupted white matter integrity may contribute to $C D$ in the early stages of psychosis, but higher GSH may indicate an intact antioxidant response mounted to neutralize the oxidative stress. Patients who mount this GSH response may ultimately experience a better treatment response, and those with a persistent illness profile may show a significant, albeit small, effect-size reduction in GSH. ${ }^{19,75}$ An alternative possibility is that higher GSH itself may be an index of excitotoxicity, because GSH is known to act as a reservoir for glutamate when normal routes of glutamate synthesis are impaired. ${ }^{76}$ Our recent fMRI-based causal modelling did not support this idea, instead favouring a protective role for GSH against glutamatergic excitotoxicity. ${ }^{77}$ Finally, a reduction in glutamate-GSH ratio reported here may indicate acute astrogliosis and/or rarefication of glutamatergic synapses. These mechanistic underpinnings could not be resolved with our current data; pharmaco-fMRI studies manipulating GSH levels will be critical for future insights.

\section{Limitations}

Our study had notable strengths, including the use of highly resolved $7 \mathrm{~T}$ spectroscopy (which allowed for concurrent GSH and glutamate quantification) a combined DTI-MRS protocol and the recruitment of minimally medicated patients with FEP.

Several limitations must be considered as well. First, because we optimized our acquisition for concurrent measurement of 
glutamate and GSH, glutamine spectra were estimated with higher uncertainty (Appendix 1). Second, we relied on a single voxel placed at the dorsal ACC, in line with other $7 \mathrm{~T}$ MRS studies in psychosis; ${ }^{78,79}$ the status of GSH and glutamate in relation to $\mathrm{CD}$ may differ in other brain regions. We also restricted our DTI analysis to the cingulum, given the previous work of Bopp and colleagues. ${ }^{7}$ A TBSS analysis across the entire white matter skeleton (reported in Appendix 1) revealed no significant group differences. Although we had sufficient power to detect a group difference between the high and low CD groups in a hypothesis-driven search restricted to a single tract, our sample was likely of insufficient size for a whole brain mass univariate search with necessary corrections for multiple testing.

\section{Conclusion}

The neural correlates of acute conceptual disorganization likely relate to cortical oxidative stress and disrupted white matter integrity. Given the functional importance of persistent disorganization, we call for clinical trials of antioxidants and agents that improve neuroglial interactions to focus on addressing the burden of disorganization in psychosis.

Affiliations: From the Department of Psychiatry, Second Xiangya Hospital of Central South University, Changsha, Hunan, China (Pan); the Robarts Research Institute, University of Western Ontario, London, Ont., Canada (Pan, Khan, Palaniyappan); the Lawson Health Research Institute, London, Ont., Canada (Théberge, Palaniyappan); the Department of Medical Biophysics, University of Western Ontario, London, Ont., Canada (Jeon, Théberge, Khan, Palaniyappan); the Department of Psychiatry, University of Western Ontario, London, Ont., Canada (Palaniyappan, Théberge); the Department of Psychiatry, Dalhousie University, Halifax, NS, Canada (Dempster); the China National Clinical Research Center on Mental Disorders (Xiangya), Changsha, Hunan, China (Pan); the China National Technology Institute on Mental Disorders, Changsha, Hunan, China (Pan); the Hunan Key Laboratory of Psychiatry and Mental Health, Changsha, Hunan, China (Pan); and the Institute of Mental Health of Second Xiangya Hospital, Central South University, Changsha, Hunan, China (Pan).

Funding: This study was funded by CIHR Foundation Grant No. 375104/2017 (to LP); Schulich School of Medicine Clinical Investigator Fellowship (to KD); graduate student salary support of PJ from NSERC Discovery Grant No. RGPIN2016-05055 (to JT); Canada Graduate Scholarship (to KD); the National Natural Science Foundation of China, grant numbers 81671335, 81701325, 81801353 (to YP). Data acquisition was supported by the Canada First Excellence Research Fund to BrainSCAN, Western University (Imaging Core); Innovation fund for Academic Medical Organization of Southwest Ontario; Bucke Family Fund, the Chrysalis Foundation and the Arcangelo Rea Family Foundation (London, Ontario).

Competing interests: L. Palaniyappan reports grants and personal fees from Otsuka Canada, grants and personal fees from Janssen Canada, grants from Sunovion, personal fees from SPMM Course (United Kingdom), other fees from Oxford University Press and personal fees from the Canadian Psychiatric Association, all outside the submitted work. He is an associate editor of $J P N$, but was not involved in the decision to accept this manuscript for publication. No other competing interests were declared.

Contributors: L. Palaniyappan designed the study. K. Dempster and J. Théberge acquired the data, which Y. Pan, P. Jeon and A. Khan analyzed. Y. Pan and L. Palaniyappan wrote the article, which K. Dempster, P. Jeon, J. Théberge and A. Khan reviewed. All authors approved the final version to be published and can certify that no other individuals not listed as authors have made substantial contributions to the paper.

Content licence: This is an Open Access article distributed in accordance with the terms of the Creative Commons Attribution (CC BYNC-ND 4.0) licence, which permits use, distribution and reproduction in any medium, provided that the original publication is properly cited, the use is noncommercial (i.e., research or educational use), and no modifications or adaptations are made. See: https:/ / creativecommons.org/licenses/by-nc-nd/4.0/

\section{References}

1. Minor KS, Marggraf MP, Davis BJ, et al. Conceptual disorganization weakens links in cognitive pathways: disentangling neurocognition, social cognition, and metacognition in schizophrenia. Schizophr Res 2015;169:153-8.

2. Rocca P, Galderisi S, Rossi A, et al. Disorganization and real-world functioning in schizophrenia: results from the multicenter study of the Italian Network for Research on Psychoses. Schizophr Res 2018;201:105-12.

3. Minor KS, Lysaker PH. Necessary, but not sufficient: links between neurocognition, social cognition, and metacognition in schizophrenia are moderated by disorganized symptoms. Schizophr Res 2014;159:198-204.

4. Minor KS, Marggraf MP, Davis BJ, et al. Conceptual disorganization weakens links in cognitive pathways: disentangling neurocognition, social cognition, and metacognition in schizophrenia. Schizophr Res 2015;169:153-8.

5. Viher PV, Stegmayer K, Giezendanner S, et al. Cerebral white matter structure is associated with DSM-5 schizophrenia symptom dimensions. Neuroimage Clin 2016;12:93-9.

6. Cavelti M, Winkelbeiner S, Federspiel A, et al. Formal thought disorder is related to aberrations in language-related white matter tracts in patients with schizophrenia. Psychiatry Res Neuroimaging 2018;279:40-50.

7. Bopp MHA, Zollner R, Jansen A, et al. White matter integrity and symptom dimensions of schizophrenia: a diffusion tensor imaging study. Schizophr Res 2017;184:59-68.

8. de Boer JN, van Hoogdalem M, Mandl R, et al. Language in schizophrenia: relation with diagnosis, symptomatology and white matter tracts. NPJ Schizophr 2020;6:10.

9. Barth C, Lonning V, Gurholt TP, et al. Exploring white matter microstructure and the impact of antipsychotics in adolescent-onset psychosis. PLoS One 2020;15:e0233684.

10. Voineskos AN, Mulsant BH, Dickie EW, et al. Effects of antipsychotic medication on brain structure in patients with major depressive disorder and psychotic features: neuroimaging findings in the context of a randomized placebo-controlled clinical trial. JAMA Psychiatry 2020;77:674-83.

11. Kircher T, Bröhl H, Meier F, et al. Formal thought disorders: from phenomenology to neurobiology. Lancet Psychiatry 2018;5:515-26.

12. Cavelti M, Kircher T, Nagels A, et al. Is formal thought disorder in schizophrenia related to structural and functional aberrations in the language network? A systematic review of neuroimaging findings. Schizophr Res 2018;199:2-16.

13. Adler CM, Malhotra A, Elman I, et al. Comparison of ketamineinduced thought disorder in healthy volunteers and thought disorder in schizophrenia. Am J Psychiatry 1999;156:1646-9.

14. Do KQ, Cabungcal JH, Frank A, et al. Redox dysregulation, neurodevelopment, and schizophrenia. Curr Opin Neurobiol 2009;19:220-30.

15. Baxter PS, Hardingham G. Adaptive regulation of the brain's antioxidant defences by neurons and astrocytes. Free Radic Biol Med 2016;100:147-52

16. Alameda L, Fournier M, Khadimallah I, et al. Redox dysregulation as a link between childhood trauma and psychopathological and neurocognitive profile in patients with early psychosis. Proc Natl Acad Sci U S A 2018;115:12495-500.

17. Zhang XY, Tan Y, Cao L, et al. Antioxidant enzymes and lipid peroxidation in different forms of schizophrenia treated with typical and atypical antipsychotics. Schizophr Res 2006;81:291-300.

18. Chien YL, Hwu H, Hwang T, et al. Clinical implications of oxidative stress in schizophrenia: acute relapse and chronic stable phase. Prog Neuropsychopharmacol Biol Psychiatry 2020;99:109868.

19. Das TK, Javadzadeh A, Dey A, et al. Antioxidant defense in schizophrenia and bipolar disorder: a meta-analysis of MRS studies of anterior cingulate glutathione. Prog Neuropsychopharmacol Biol Psychiatry 2019;91:94-102. 
20. Sydnor VI, Roalf DR. A meta-analysis of ultra-high field glutamate, glutamine, GABA and glutathione ${ }^{1} \mathrm{HMRS}$ in psychosis: implications for studies of psychosis risk. Schizophr Res 2020;226:61-9.

21. Domen P, Peeters S, Michielse S, et al. Differential time course of microstructural white matter in patients with psychotic disorder and individuals at risk: a 3-year follow-up study. Schizophr Bull 2017;43:160-70.

22. Keymer-Gausset A, Alonso-Solis A, Corripio I, et al. Gray and white matter changes and their relation to illness trajectory in first episode psychosis. Eur Neuropsychopharmacol 2018;28:392-400.

23. Fujiwara $\mathrm{H}$, Namiki $\mathrm{C}$, Hirao K, et al. Anterior and posterior cingulum abnormalities and their association with psychopathology in schizophrenia: a diffusion tensor imaging study. Schizophr Res 2007:95:215-22.

24. Palaniyappan L, Al-Radaideh A, Mougin O, et al. Aberrant myelination of the cingulum and Schneiderian delusions in schizophrenia: a 7T magnetization transfer study. Psychol Med 2019; 49:1890-6.

25. Wong HJ, Chew $\mathrm{QH}$, Lee $\mathrm{RD}$, et al. Illness remission status and commissural and associative brain white matter fiber changes in schizophrenia. PsyCh J 2020;9:894-902.

26. Fitzsimmons J, Rosa P, Sydnor VJ, et al. Cingulum bundle abnormalities and risk for schizophrenia. Schizophr Res 2020;215:385-91.

27. Smucny J, Barch DM, Gold JM, et al. Cross-diagnostic analysis of cognitive control in mental illness: insights from the CNTRACS consortium. Schizophr Res 2019;208:377-83.

28. Nestor PG, Kubicki M, Spencer KM, et al. Attentional networks and cingulum bundle in chronic schizophrenia. Schizophr Res 2007:90:308-15.

29. Tyburski E, Mak M, Samochowiec A, et al. The relationship between cingulum bundle integrity and different aspects of executive functions in chronic schizophrenia. Prog Neuropsychopharmacol Biol Psychiatry 2020;102:109955.

30. Metzler-Baddeley C, Jones D, Steventon J, et al. Cingulum microstructure predicts cognitive control in older age and mild cognitive impairment. J Neurosci 2012;32:17612-9.

31. Palaniyappan L, Mahmood J, Balain V, et al. Structural correlates of formal thought disorder in schizophrenia: an ultra-high field multivariate morphometry study. Schizophr Res 2015;168:305-12.

32. Palaniyappan L, Al-Radaideh A, Gowland P, et al. Cortical thickness and formal thought disorder in schizophrenia: an ultra highfield network-based morphometry study. Prog Neuropsychopharmacol Biol Psychiatry 2020;101:109911.

33. Bathelt J, Johnson A, Zhang M, et al. The cingulum as a marker of individual differences in neurocognitive development. Sci Rep 2019;9:2281

34. Monin A, Baumann PS, Griffa A, et al. Glutathione deficit impairs myelin maturation: relevance for white matter integrity in schizophrenia patients. Mol Psychiatry 2015;20:827-38.

35. Leckman JF, Sholomskas D, Thompson WD, et al. Best estimate of lifetime psychiatric-diagnosis-a methodological study. Arch Gen Psychiatry 1982;39:879-83.

36. Kocsis RN. Diagnostic and statistical manual of mental disorders: fifth edition (DSM-5). Int J Offender Ther Comp Criminol 2013;57:1546-8.

37. Remington G, Addington D, Honer W, et al. Guidelines for the pharmacotherapy of schizophrenia in adults. Can J Psychiatry 2017;62:604-16.

38. Limongi R, Mackinley M, Dempster K, et al. Frontal-striatal connectivity and positive symptoms of schizophrenia: implications for the mechanistic basis of prefrontal rTMS. Eur Arch Psychiatry Clin Neurosci 2020;doi: 10.1007/s00406-020-01163-6.

39. Dempster K, Jeon P, MacKinley M, et al. Early treatment response in first episode psychosis: a 7-T magnetic resonance spectroscopic study of glutathione and glutamate. Mol Psychiatry 2020;25:1640-50.

40. Lin $\mathrm{CH}$, Lin HS, Lin SC, et al. Early improvement in PANSS-30, PANSS-8, and PANSS-6 scores predicts ultimate response and remission during acute treatment of schizophrenia. Acta Psychiatr Scand 2018;137:98-108.

41. Morosini PL, Magliano L, Brambilla L, et al. Development, reliability and acceptability of a new version of the DSM-IV Social and Occupational Functioning Assessment Scale (SOFAS) to assess routine social funtioning. Acta Psychiatr Scand 2000;101:323-9.

42. Dickinson D, Ragland JD, Gold JM, et al. General and specific cognitive deficits in schizophrenia: Goliath defeats David? Biol Psychiatry 2008;64:823-7.
43. Palaniyappan L, Al-Radaideh A, Mougin O, et al. Combined white matter imaging suggests myelination defects in visual processing regions in schizophrenia. Neuropsychopharmacology 2013;38:1808-15.

44. Mori S, Wakana S, van Zijl PCM, et al. MRI atlas of human white matter. 1st ed. Amsterdam, Netherlands: Elsevier; 2005

45. Andreasen NC, Carpenter W, Kane J, et al. Remission in schizophrenia: proposed criteria and rationale for consensus. Am J Psychiatry 2005;162:441-9.

46. Çokal D, Sevilla G, Jones W, et al. The language profile of formal thought disorder. NPJ Schizophr 2018;4:18

47. Jenkinson M, Beckmann CF, Behrens TE, et al. FSL. NeuroImage 2012;62:782-90.

48. Gorgolewski KJ, Auer T, Calhoun VD, et al. The brain imaging data structure, a format for organizing and describing outputs of neuroimaging experiments. Sci Data 2016;3:160044

49. Gorgolewski KJ, Alfaro-Almagro F, Auer T, et al. BIDS apps: improving ease of use, accessibility, and reproducibility of neuroimaging data analysis methods. PLOS Comput Biol 2017;13;e1005209.

50. Veraart J, Novikov DS, Christiaens D, et al. Denoising of diffusion MRI using random matrix theory. Neuroimage 2016;142:394-406.

51. Kellner E, Dhital B, Kiselev VG, et al. Gibbs-Ringing artifact removal based on local subvoxel-shifts. Magn Reson Med 2016;76:1574-81.

52. Andersson JLR, Skare S, Ashburner J. How to correct susceptibility distortions in spin-echo echo-planar images: application to diffusion tensor imaging. Neuroimage 2003;20:870-88.

53. Andersson JLR, Sotiropoulos SN. An integrated approach to correction for off-resonance effects and subject movement in diffusion MR imaging. Neuroimage 2016;125:1063-78.

54. Andersson JLR, Graham MS, Zsoldos E, et al. Incorporating outlier detection and replacement into a non-parametric framework for movement and distortion correction of diffusion MR images. Neuroimage 2016;141:556-72.

55. Modat M, Cash D, Daga P, et al. Global image registration using a symmetric block-matching approach. J Med Imaging (Bellingham) 2014;1:024003.

56. Jovicich J, Czanner S, Greve D, et al. Reliability in multi-site structural MRI studies: effects of gradient non-linearity correction on phantom and human data. Neuroimage 2006;30:436-43.

57. Jenkinson M, Beckmann CF, Behrens TE, et al. Fsl. Neuroimage 2012;62:782-90

58. Kumar J, Liddle EB, Fernandes CC, et al. Glutathione and glutamate in schizophrenia: a 7T MRS study. Mol Psychiatry 2020;25:873-82.

59. Near J, Edden R, Evans CJ, et al. Frequency and phase drift correction of magnetic resonance spectroscopy data by spectral registration in the time domain. Magn Reson Med 2015;73:44-50.

60. Stanley JA, Drost DJ, Williamson PC, et al. The use of a-priori knowledge to quantify short echo in-vivo H-1 MR spectra. Magn Reson Med 1995;34:17-24

61. Wong D, Schranz A, Bartha R. Optimized in vivo brain glutamate measurement using long-echo-time semi-LASER at 7 T. NMR Biomed 2018;31:e4002

62. Dey A, Dempster K, Mackinley M, et al. Conceptual disorganization and redistribution of resting-state cortical hubs in untreated first-episode psychosis: a 7T study. NPJ Schizophr 2021;7:4.

63. Winkler AM, Ridgway GR, Webster MA, et al. Permutation inference for the general linear model. Neuroimage 2014;92:381-97.

64. Smith SM, Nichols TE. Threshold-free cluster enhancement: addressing problems of smoothing, threshold dependence and localisation in cluster inference. Neuroimage 2009;44:83-98

65. Duarte JM, Xin L. Magnetic resonance spectroscopy in schizophrenia: evidence for glutamatergic dysfunction and impaired energy metabolism. Neurochem Res 2019;44:102-16.

66. Harris JL, Choi I, Brooks W. Probing astrocyte metabolism in vivo: proton magnetic resonance spectroscopy in the injured and aging brain. Front Aging Neurosci 2015;7:202.

67. Marques TR, Taylor $\mathrm{H}, \mathrm{Chaddock} \mathrm{C}$, et al. White matter integrity as a predictor of response to treatment in first episode psychosis. Brain 2014;137:172-82.

68. Xiao Y, Sun HQ, Shi SL, et al. White matter abnormalities in nevertreated patients with long-term schizophrenia. Am J Psychiatry 2018:175:1129-36

69. Xiang $B$, Wang $Q$, Lei $W$, et al. Genes in immune pathways associated with abnormal white matter integrity in first-episode and treatment-naive patients with schizophrenia. Br J Psychiatry 2019; 214:281-7. 
70. Ruan H, Luo Q, Palaniyappan L, et al. Topographic diversity of structural connectivity in schizophrenia. Schizophr Res 2020; 215:181-9.

71. Bubb EJ, Metzler-Baddeley C, Aggleton J. The cingulum bundle: anatomy, function, and dysfunction. Neurosci Biobehav Rev 2018;92:104-27.

72. Kubicki M, Niznikiewicz M, Connor E, et al. Relationship between white matter integrity, attention, and memory in schizophrenia: a diffusion tensor imaging study. Brain Imaging Behav 2009;3: 191-201

73. Takei K, Yamasue H, Abe O, et al. Structural disruption of the dorsal cingulum bundle is associated with impaired Stroop performance in patients with schizophrenia. Schizophr Res 2009;114:119-27.

74. Demro C, Rowland L, Wijtenburg S, et al. Glutamatergic metabolites among adolescents at risk for psychosis. Psychiatry Res 2017; 257:179-85.
75. Kumar J, Liddle E, Fernandes C, et al. Glutathione and glutamate in schizophrenia: a 7T MRS study. Mol Psychiatry 2020;25:873-82.

76. Sedlak TW, Paul B, Parker G, et al. The glutathione cycle shapes synaptic glutamate activity. Proc Natl Acad Sci U S A 2019;116:2701-6.

77. Limongi $\mathrm{R}$, Jeon $\mathrm{P}$, Théberge J, et al. Counteracting effects of glutathione on the glutamate-driven excitation/inhibition imbalance in first-episode schizophrenia: a 7T MRS and dynamic causal modeling study. Antioxidants (Basel, Switzerland) 2021;10(1).

78. Overbeek G, Gawne T, Reid M, et al. Relationship between cortical excitation and inhibition and task-induced activation and deactivation: a combined magnetic resonance spectroscopy and functional magnetic resonance imaging study at 7T in first-episode psychosis. Biol Psychiatry Cogn Neurosci Neuroimaging 2019;4:121-30.

79. Rowland LM, Pradhan S, Korenic S, et al. Elevated brain lactate in schizophrenia: a $7 \mathrm{~T}$ magnetic resonance spectroscopy study. Transl Psychiatry 2016;6:e967. 